\title{
PLURAL: ATUAÇÃO DINÂMICA E TRANSVERSAL DO GESTOR CULTURAL
}

Ludmila Ferreira Ribeiro

\begin{abstract}
Especializada em Gestão Cultural e Organização de Eventos da Escola de Comunicação e Cultura. Pesquisa orientada pelo Prof. Dr. Juarez Tadeu de Paula Xavier e pelo Prof. José Márcio Barros.
\end{abstract}

\section{Resumo}

O presente artigo tem como proposta refletir sobre o processo de profissionalização do campo da cultura, considerando a importância da área para o desenvolvimento sustentável e a necessidade de reunir na prática técnicas e conhecimentos transversais que favoreçam a dinâmica de atuação dos profissionais na cadeia produtiva da cultura. Tendo como base a produção contemporânea especificamente sobre a organização da cultura, gestão e produção e a formação dos profissionais da área, são apresentados desafios e responsabilidades do gestor cultural em contexto de globalização, diversidade cultural e estruturação de políticas públicas para a cultura.

Palavras-chave: Gestão; cultura; cidadania; profissionalização

\section{Resumen}

El presente trabajo trae como propuesta la reflexión a cerca de la necesidad de profesionalización en el campo cultural, llevándose en cuenta la importancia del tema para el desarrollo sustentable, la necesidad de reunir en la práctica las técnicas y conocimientos transversales que favorezcan la dinámica actuación de los profesionales en la cadena de producción cultural. Basados en la producción contemporánea, de forma específica sobre la organización de la cultura, gestión y producción, además de la formación de los profesionales del área, son presentadas responsabilidades y desafíos para el gestor cultural en un contexto de globalización, diversidad cultural y estructuración de políticas públicas para cultura.

Palabras clave: Gestión; cultura; ciudadanía; profesionalización

\begin{abstract}
The present article aims at reflecting upon the necessities of professionalization within culture field, taking into consideration the importance of this area for the sustainable development and the need to meet in practice technical and general skills to promote the dynamics of supply chain professionals in the culture. Based on the contemporary production specifically focused on culture organization , management and production, and also on the formation of those professionals from the area, presents challenges and responsibilities of the manager in the context of cultural globalization, cultural diversity and structuring of public policies for culture.
\end{abstract}

Keywords: Management; culture; citizenship; professionalization 


\section{Introdução}

O fortalecimento do campo da cultura ocasiona o surgimento de novas formas de organização e possibilidades de ação diferenciadas como área de atuação profissional, como fator de desenvolvimento e como princípio de cidadania. Um novo segmento de trabalho se estrutura em torno das práticas culturais, exigindo conhecimentos e técnicas transversais dos envolvidos nesta área profissional ainda em fase de definição.

A cultura se constitui "como campo social singular, que articula e inaugura instituições, profissões, linguagens, símbolos, valores e tensões”, (RUBIM, 2008, p. 47). Cada vez mais reconhecida como pilar do desenvolvimento sustentável, juntamente com a dimensão econômica, social e ambiental, a cultura passa a ocupar papel estratégico na atualidade, o que exige a estruturação e profissionalização da área, considerando sua dimensão executiva e conceitual.

A mediação entre o universo subjetivo das artes e da cultura com o mercado e as políticas públicas, requer a atuação integrada de um profissional que, conhecendo a ligação e as influências das diversas esferas societárias, possa viabilizar processos artísticos, desencadear o fortalecimento de identidades e contribuir para a superação de problemas sociais históricos.

A discussão contemporânea sobre a formação dos profissionais da cultura, especialmente do gestor e produtor cultural, tem resultado em cursos, publicações e seminários que são hoje a principal referência para quem atua e pesquisa nesta área. Baseando-se nas discussões e reflexões de profissionais e pesquisadores de Minas Gerais, Bahia e São Paulo, pretende-se pensar a atuação do gestor de projetos culturais considerando a sua esfera micro - desafios de estruturação da equipe de profissionais e demanda por métodos e técnicas para aprimorar o desenvolvimento de processos culturais; e sua interface com a esfera macro - impactos da ação cultural na sociedade, orientada por diretrizes nacionais e internacionais, especialmente no que tange à Convenção da UNESCO sobre a Proteção e a Promoção da Diversidade das Expressões Culturais e o Plano Nacional de Cultura - PNC que institui no Brasil o Sistema Nacional de Cultura - SNC.

A demanda por métodos e técnicas que possam aprimorar o fazer cultural e viabilizar a concretização de propostas e princípios conceituais da cultura é crescente no contexto atual mundial e vivenciada diariamente na esfera privada. $\mathrm{O}$ desafio é alcançar uma atuação profissional na área cultural que garanta qualidade e resultados, fundamentando essa prática em conceitos e reflexões que permitam à cultura garantir a sua interação permanente com as diversas esferas do social, do político e do econômico, não se rendendo a nenhum deles e configurando-se como uma alternati- 
va real e de convergência.

\section{Empreendendo Processos Culturais}

A construção do campo profissional da cultura requer análise permanente dos desdobramentos e potencialidades do campo mais amplo da cultura e a sistematização das práticas usuais e possíveis nesse campo. Os profissionais à frente destes processos podem contribuir de forma efetiva para a organização do setor, aliando sua referência empírica às reflexões e propostas para consolidação do campo da cultura.

A produção intelectual sobre o campo profissional da cultura é recente e está em pleno desenvolvimento, gerando inúmeras propostas e apontamentos. Intelectuais e profissionais experientes sistematizam sua prática e compartilham métodos em diversas publicações, seminários, cursos e encontros. É o caso de publicações como "O Avesso da Cena: notas sobre produção e gestão cultural”, de Romulo Avelar; "Organização e Produção da Cultura", organizado por Linda Rubim, "Gestão Cultural: profissão em formação", de Maria Helena Cunha, e o livro "Diversidade Cultural: da proteção à promoção", organizado por José Márcio Barros.

Os Anais dos ENECULT - Encontro de Estudos Multidisciplinares em Cultura, realizados em 2006, 2007 e 2008 pela Faculdade de Comunicação da Universidade Federal da Bahia, sob coordenação do Professor Albino Rubim, apresentam artigos de inúmeros pesquisadores, dentre os quais se destaca, além dos autores já citados, Albino Rubim, Enrique Saraiva, Lala Dehezelin, dentre outros. A Revista Observatório Itaú Cultural, número seis, dedicada ao tema "Os profissionais da cultura: formação para o setor cultural”, também reúne dados referenciais para pesquisa na área.

As discussões conceituais sobre cultura e sua relação com o "macrossocial, processual e público", como sugere o professor Antonio Albino Canelas Rubim, no artigo "Formação em Organização da Cultura no Brasil", são recorrentes e fundamentais para construção da práxis do gestor cultural, que precisa ter uma visão global do processo para atuar dentro da capacidade máxima do potencial da cultura em seus desdobramentos sociais, educacionais, políticos e de desenvolvimento.

Da mesma forma, na estruturação do campo da cultura é preciso diferenciar o papel dos profissionais envolvidos e agregar métodos e técnicas que contribuam para o melhor desempenho das equipes atuantes na cadeia produtiva, garantindo, por conseguinte, qualidade e fortalecimento das práticas artísticas e culturais, respeitando-se a diversidade e as especificidades de cada grupo, projeto ou instituição.

Na realização de projetos e ações culturais estão envolvidos profissionais que 
atuam nas diversas etapas de produção, circulação, distribuição e consumo que compõem a cadeia produtiva da cultura. $\mathrm{O}$ trabalho criativo dos artistas e intelectuais é central nesta cadeia, uma vez que a partir das formas de expressão artística são desencadeados outros processos, realizados por profissionais que, em outros elos da cadeia, tornam possível o exercício profissional no campo cultural.

Para atuar com esta matéria prima da ordem do subjetivo e do intangível, historicamente se envolveram pessoas que possuíam afinidade e sensibilidade com o universo artístico e buscavam de forma amadora executar as demandas burocráticas que precediam a criação e não eram de competência dos artistas. Com o crescimento do cenário cultural, a ampliação das fontes de financiamento e a estruturação de políticas públicas para o setor, são requeridos conhecimentos profissionais que tangenciem áreas como a administração, comunicação, gestão, antropologia, finanças, dentre outros. O compartilhamento de saberes e experiências nestas áreas tem sido estratégico para definição do perfil dos profissionais da cultura.

A especificidade da área cultural requer um trabalho integrado que se apropria de técnicas e conhecimentos interdisciplinares, ao mesmo tempo em que precisa desenvolver métodos próprios e específicos. Cada realização cultural, por mais que possua etapas básicas comuns de planejamento, estruturação de cronogramas, checklists, indicadores, dentre outros, apresenta peculiaridades que vão exigir inovação e criatividade dos profissionais no momento de solucionar as demandas.

Este espaço de práticas e formulações encontra-se em fase de construção o que faz com que a denominação dos profissionais envolvidos e a divisão de suas funções não estejam sedimentadas no mercado cultural.

"A pluralidade de denominações não só indica a idade recente das práticas e dos estudos acerca da organização da cultura, mas sugere pensar em itinerários e peculiaridades nacionais no desenvolvimento do tema, com forte incidência sobre a formação de seus profissionais" (RUBIM, 2008: p. 52)

As diferentes nomenclaturas e a definição das atribuições de cada um destes ofícios oscilam seja por região, por entidade e por área de expressão artística e esta questão ganha espaço nas principais instâncias de discussão contemporânea.

Publicações como dos profissionais e pesquisadores Linda Rubim, Maria Helena Cunha e Romulo Avelar buscam elucidar processos, características e práticas próprias do gestor e do produtor cultural. Estes profissionais não encontram graduação específica no Brasil e desde meados de 1980 vem experimentando uma prática intuitiva e no atual contexto precisam aprimorar competências e se profissionalizar para atuar no cenário de leis de incentivo, economia criativa e busca por políticas públicas para a cultura. 
Especialmente a publicação "O Avesso da Cena", de Romulo Avelar, detalha as etapas do trabalho no campo profissional da cultura, que envolve desde elaboração de projetos e captação de recursos, passando pela pré-produção e execução das ações, até a estruturação de relatórios e prestação de contas. Sendo um compartilhamento de sua experiência profissional a frente de grupos e projetos consolidados, "O Avesso da Cena" traz referências de sistematização e exemplos de ferramentas de planejamento, monitoramento e avaliação, propondo uma aproximação da gestão cultural com as práticas de administração de empresas, como forma de potencializar este recente ofício.

A diferenciação entre as atribuições do produtor e do gestor de projetos culturais é fundamental para se aproximar do perfil esperado destes profissionais, discriminando suas responsabilidades e demandas de ferramentas de trabalho.

Entre os estudiosos da área, parece ser um consenso à diferenciação entre estes dois profissionais, sendo o produtor ligado "a projetos de caráter mais eventual e microssocial" e o gestor atuando com projetos culturais mais permanentes, processuais e amplos. (RUBIM, 2008, p. 54). O produtor é o agente diretamente envolvido e responsável pela realização de eventos e projetos culturais, garantindo a qualidade e a concretização do produto cultural, executando para isso todas as demandas e imprevistos resultantes da produção. (AVELAR, 2008, p. 52). O gestor por outro lado tem uma atribuição que antecede e precede o trabalho do produtor, uma vez que cabe a este profissional atuar em rede e planejar, avaliar as ações em todos os contextos, macro, micro e nano, criando oportunidades para o futuro. (AVELAR, 2008, p. 54).

Ambos os profissionais estão diretamente ligados às etapas da cadeia produtiva da cultura, desempenhando papéis diferentes e complementares, sendo "imprescindível afirmar a pertença das duas noções ao registro de organização da cultura e, por conseguinte, a inevitável proximidade entre elas" (RUBIM, 2008, p. 54). No entanto, este artigo opta por focar no papel do gestor de projetos culturais, considerando a maior abrangência de sua atuação e sua capacidade de intervenção nas outras áreas de interface.

O gestor de projetos culturais tem uma atuação permanente, complexa e transversal, desenvolvendo "programas e atividades essenciais ao funcionamento de grupos, empresas e instituições ligadas ao fazer cultural.” (AVELAR, 2008, p. 55). Seu trabalho não se inicia, nem mesmo se esgota na realização de uma ação, seja um evento, um espetáculo ou um seminário e tem impactos de longo prazo. $\mathrm{O}$ trabalho de estruturação da proposta artística e elaboração de um projeto cultural; a avaliação dos possíveis meios de incentivo e financiamento; o planejamento de estratégias de ação para garantir sucesso em todas as etapas do trabalho; a coordenação da equipe e da divulgação das ações; o atendimento de demandas dos stakeholders; a reestru- 
turação das metas e procedimentos sempre que necessário e a avaliação e relatoria das ações realizadas visando aprimoramento e continuidade; todas essas atribuições fazem parte do escopo de atuação do gestor, responsável ainda pela contratação e coordenação das equipes indispensáveis ao cumprimento de todas as etapas.

A visão global é indispensável ao gestor, que precisa ter conhecimento aprofundado da cadeia produtiva, das etapas da ação cultural e dos conceitos interdisciplinares que permeiam a sua atuação, como apresentam Cunha e Bertelli (2008):

\footnotetext{
"O gestor da cultura lida com temáticas complexas e amplas. Desde as tecnologias digitais voltadas para a produção e comercialização de produtos e serviços das chamadas indústrias criativas, as diferentes linguagens artísticas, seus recursos humanos, políticas específicas e aquelas que afetam diretamente o campo da cultura, até aspectos da economia, do direito, da comunicação e dos programas de desenvolvimento dos territórios em que as práticas culturais são decisivas para seus resultados".
}

Na etapa executiva do trabalho do gestor, faz-se necessário estruturar e definir técnicas de gerenciamento e administração que possam potencializar e ampliar o desempenho dos profissionais da cultura neste contexto complexo e em permanente transformação. Avelar (2008) relaciona as atividades típicas do administrador, "a quem compete atingir determinados objetivos a partir do desempenho de cinco atividades: prever, organizar, comandar, coordenar e controlar", com a dinâmica dos gestores culturais que, se apropriando desta lógica, poderão ter condições de otimizar e aprimorar o desempenho profissional no campo da cultura.

Além destes princípios da administração, outros recursos da área são citados pelo autor como fonte de referência para que as práticas culturais sejam fundamentadas em resultado e qualidade e possam garantir a sua sustentabilidade. Dentre estes recursos destaca-se a estruturação de plano de negócios; a construção de planejamento estratégico e plano de ação para orientar as práticas da instituição ou grupo cultural a curto e médio prazo; a definição da estrutura organizacional e a meta por qualidade e excelência nos produtos e serviços culturais ofertados.

Saraiva (2008) sintetiza o papel do gestor cultural, considerando sua atribuição "implementar normas, planos e projetos, estabelecer estruturas, alocar recursos humanos, financeiros, físicos e tecnológicos e, principalmente, empenhar criatividade e capacidade de inovação para atingir esses objetivos da melhor forma possível”.

Detectar as diversas formas de interação entre o fenômeno cultural e o fenômeno organizacional é um investimento que precisa ser feito principalmente nos programas de formação dos profissionais da cultura, visando disponibilizar ao administrador cultural instrumentos aplicáveis à diversidade de frentes de atuação. 
O desafio é promover a aproximação do campo profissional da cultura com as técnicas de administração de forma ética e sustentável, considerando que as ferramentas de trabalho devem potencializar o trabalho do gestor na mediação das artes como o mundo, buscando "os pontos de convergência que existem entre as várias linguagens” (DEHEIZELIN, 2006) que compõe a área cultural, explorando as potencialidades da cultura de garantir resultados além da realização do projeto.

A introdução da lógica de administração que dialoga com a política, com a economia e com o mercado, deve ter como finalidade a mediação necessária da arte e da cultura no mundo global, consolidando o seu espaço estratégico e não apenas servindo de ferramenta para interesses específicos.

Em entrevista para a Revista Observatório Itaú Cultural, Barros (2008) coloca que o avanço no campo profissional da cultura se deu "menos pela perspectiva do bem público e dos compromissos com a cidadania, do que pela pressão do mercado alimentado pelos mecanismos de patrocínio cultural e incentivo fiscal". A demanda crescente do mercado, especialmente nas relações da cultura com o marketing e as leis de incentivo exige a organização da área, que, entretanto não pode gerar "uma apropriação mecânica e pouco crítica de ferramentas da área gerencial, desprovidas de conceitos e teorias". (BARROS, 2008).

"A cultura como pilar do desenvolvimento sustentável, juntamente com a dimensão econômica e social" (FÓRUM MUNDIAL U-40, 2009) é dado imperativo no contexto atual e este espaço precisa ser ocupado por criadores e gestores fundamentados em referências conceituais e reflexivas que possam contribuir por meio da dimensão artística e cultural para o desenvolvimento humano e fortalecimento de identidades em meio à diversidade.

O gestor cultural munido de ferramentas deve saber recriá-las e colocá-las a serviço da criação, viabilizando sua expressividade e autenticidade e promovendo o diálogo desta forma de expressão com outras afins e com o seu público. Conhecer o universo artístico e a diversidade cultural é fundamental para comunicar e circular a criação no contexto global. Encontrar meios para a cultura, considerando suas especificidades, é criar alternativas para a lógica saturada do mercado que não comporta a complexidade e dinâmica dos processos culturais e nem por isso o inviabiliza.

O cenário profissional se amplia, tornando-se também mais complexo, uma vez que a cultura se configura como espaço de "convergência de interesses governamentais, multilaterais e empresariais, respaldada por vasta gama de iniciativas do terceiro setor" (DEHEIZELIN, 2006) e as manifestações artísticas se fortalecem em diversas esferas, facilitadas pelas novas tecnologias, representando identidades e demandas de diferentes grupos. 
O fortalecimento da cena artística independente e a valorização de manifestações culturais tradicionais, populares e contemporâneas na diversidade cultural, compõem um novo cenário não mais submetido apenas à lógica do mercado. A cultura consolida-se como um campo societário de interface com diversas áreas e com espaços de articulação, lógicas e demandas próprias. Neste cenário se configura o perfil do gestor cultural, atuando no gerenciamento de processos, recursos e pessoas, desafiado a criar novos espaços de difusão e circulação da cultural e participar da construção e consolidação de políticas e diretrizes para o setor.

Diante da amplitude e alcance da cultura, cresce a responsabilidade de gestores culturais incumbidos de garantir a execução das dinâmicas culturais sejam eventos, projetos, ações de formação, espetáculos. Os resultados alcançados em outras esferas são desdobramentos possíveis da prática cultural e devem ser considerados estratégicos neste contexto de crise de hegemonia e de busca por alternativas. Desta feita, um impacto da ação cultural é o estímulo aos cidadãos no desenvolvimento e uso de suas capacidades para solucionar problemas comuns em âmbito particular e coletivo.

\footnotetext{
"A energia política vital e as candentes questões de uma nação serão encontradas, com mais freqüência, dentro e no entorno dos movimentos sociais, não nas instituições oficiais da democracia”. (DOWNING, 2004: p. 58).
}

A atuação profissional no campo da cultura considerando a dimensão da práxis pode fortalecer e estimular práticas tradicionais e o surgimento de outras formas emergentes de criação que expressem anseios e propostas de grupos sociais resistentes neste cenário de “dominação e subordinação” (HALL, 2003, p. 254).

\section{Novos Desafios para o Gestor da Cultura}

Compreender os processos próprios das dinâmicas culturais e investir no fortalecimento das identidades em um contexto de diversidade cultural torna-se princípio básico para a interação dos profissionais da cultura na globalização, dispondo dos recursos da tecnologia e das redes de comunicação, sem serem suprimidos pelas lógicas hegemônica do capital.

O fortalecimento das experiências culturais, envolvendo as manifestações artísticas e populares e todas as formas de expressão, hábitos e vivências dos grupos sociais, favorece a construção de espaços de produção de sentido, de valorização de identidades e de desenvolvimento humano. Sendo "uma espécie de campo de batalha permanente, (onde) há um luta contínua e necessariamente irregular e desigual, por parte da cultura dominante, no sentido de desorganizar e reorganizar constantemente a cultura popular" (HALL, 2003, p. 255) a cultura ocupa papel estratégico na sociedade, podendo se configurar tanto como meio de dominação e controle de grupos, 
ou no extremo oposto, como forma de resistência, de expressão e de proposição.

Estes extremos podem ser exemplificados pela indústria cultural e a diversidade cultural como princípio universal e são parte constitutiva do cenário de atuação do profissional da cultura. Lidar com as contradições e as potencialidades da cultura requer destes profissionais análises críticas sobre os padrões hegemônicos e as possibilidades de transformação.

Bases para a atuação crítica e consciente estão propostas em documentos de relevância internacional, como a Convenção da UNESCO sobre a Proteção e Promoção da Diversidade das Expressões Culturais; e em âmbito nacional, no Plano Nacional de Cultura, que orientará a política pública de cultura no Brasil nos próximos 10 anos e institui o Sistema Nacional de Cultura. Estes documentos representam desafios para o gestor ao apresentar diretrizes e propostas conceituais que precisam ser incorporadas às políticas e dinâmicas culturais em âmbito global e local.

\footnotetext{
"Ao se falar de diversidade cultural nos referimos a modelos normativos diversos que ordenam não apenas a produção e as trocas simbólicas no campo estético, religioso e lúdico, mas que se referem também às maneiras como se definem as formas de aprendizagem, circulação, apropriação, distribuição, mercantilização de bens e processos culturais" (BARROS, 2008: p. 02).
}

No momento em que a cultura passa a ocupar lugar estratégico nas diretrizes nacionais e internacionais, cabe o gestor, principal interlocutor entre estas diversas esferas, conhecer, difundir e apresentar propostas que considerem a aplicação efetiva destas diretrizes. Barros (2008) problematiza a "tensão crítica entre modelos culturais e gerenciais", considerando a complexidade do campo da diversidade cultural que não se restringe às diferenças estéticas, mas remete também aos "modos de gestão que se fazem presentes nos diferentes padrões culturais”. (BARROS, 2008, p. 2).

É fundamental considerar esta tensão no momento de se propor a inclusão de ferramentas inteligentes da administração no campo da cultura. A profissionalização dos agentes envolvidos no processo cultural não pode resultar em padronização e engessamento. Devem ser desenvolvidas metodologias e ferramentas considerando a possibilidade de democratização e recriação das mesmas, para adaptá-las às particularidades de cada contexto e servir para a consolidação do campo da cultura como "firme e permanente prioridade de governança mundial." (FÓRUM MUNDIAL U-40, 2009).

\footnotetext{
"Tanto no setor público, governamental e não-governamental, quanto no setor privado, emerge, mesmo que de forma ainda tímida e heterogênea, uma consciência da necessidade de se dotar o trabalho com e na cultura, de patamares de competência gerencial, compatíveis com sua importância estratégica nos campos da política, da economia, da educação, da saúde, etc". (BARROS, 2007: p. 1).
} 
O entendimento da cultura como elemento central na vida contemporânea por parte das instâncias governamentais e representativas tem resultado na estruturação de documentos e programas oficiais que, construídos e compartilhados com a atuação de organizações públicas, privadas e da sociedade civil, pretendem traçar diretrizes e garantir a efetividade das práticas culturais.

Neste sentido, conhecer, ratificar, propor e aplicar os princípios presentes na Convenção da Diversidade Cultural e no Plano Nacional de Cultura é uma forma de fundamentar práticas e consolidar o campo estratégico da cultura. A aplicação destes princípios pode ser efetivamente alcançada com a estruturação e profissionalização da área, sendo relevante o papel do gestor neste processo.

Instituída oficialmente em âmbito mundial no ano de 2005, tendo o Brasil como um dos principais países signatários, a Convenção da UNESCO sobre a Proteção e Promoção da Diversidade das Expressões Culturais legitima a diversidade cultural como "patrimônio comum da humanidade" e requer sua defesa como "um imperativo ético inseparável do respeito à dignidade da pessoa humana". (UNESCO, 2005). Os princípios da Convenção consideram que a Diversidade Cultural e o respeito às diferenças e a promoção do diálogo intercultural é garantia de equilíbrio e paz mundial. É fator de desenvolvimento, promove a autonomia dos Estados de preservar sua diversidade e identidade sem sofrer sanções e cria condições propícias para a produção e a difusão de bens e serviços culturais diversificados, ampliando a possibilidade de escolha a todos.

A ativa participação do Brasil na aprovação da Convenção da Diversidade foi concomitante ao processo de estruturação das propostas de política pública para a cultura brasileira, sendo iniciado na Gestão de Gilberto Gil e tendo hoje Juca Ferreira à frente do Ministério da Cultura no Governo Lula 2002-2010. Dentre estas propostas destaca-se a implantação do Plano Nacional de Cultura, que:

\footnotetext{
"Representará um marco de regulação de longo prazo das políticas públicas do setor em todo o País. Em seus 10 anos de duração, deverá englobar e indicar parâmetros para realizações de ações de médio e curto prazo (...) e a execução orçamentária anual de programas e ações dos poderes executivos da União, estados, Distrito Federal e municípios". (PLANO..., 2008: p. 27).
}

Em consonância com as discussões internacionais, o Plano Nacional de Cultura (PNC) se volta para a realidade brasileira. Considerando a diversidade cultural como fator indissociável da identidade do país, o PNC propõe diretrizes que garantam o pluralismo e uma maior igualdade de oportunidades, de forma que "a gestão pública ultrapasse o alcance tradicional e restritivo das belas-artes e dos produtos da indústria cultural”. (PLANO, 2008, p. 30).

O Plano Nacional de Cultura é resultado de um processo de diagnóstico, dis- 
cussão e propostas para o contexto brasileiro, que realizou a I Conferência Nacional de Cultura, em 2005, com a promoção de encontros municipais, intermunicipais, estaduais e setoriais e ampla discussão com a participação do estado e sociedade civil. Em fase de aprovação na Câmara dos Deputados e no Congresso Nacional, o PNC tem potencial de fortalecer e consolidar o cenário cultural brasileiro em âmbito internacional, sendo uma proposta de política pública para o Brasil que, com histórico de "ausência, autoritarismo e instabilidade" (RUBIM, 2008, p. 200), está pela primeira vez definindo os rumos do desenvolvimento da cultura de forma consistente e compartilhada.

O Sistema Nacional de Cultura (SNC), instituído pelo PNC será uma ferramenta de execução, acompanhamento e avaliação do Plano. A ser implementado em âmbito federal, estadual e municipal, o SNC tem como objetivo articular e integrar políticas públicas de cultura para serem pactuadas entre os entes da federação e a sociedade civil de forma a garantir "eficiência, eficácia, eqüidade e efetividade na aplicação dos recursos públicos” (SNC, 2009, p. 17), visando também a consolidação e continuidade desta política.

O Plano Nacional de Cultura e o Sistema Nacional de Cultura são formas de dar institucionalidade e organicidade às propostas de reorganização da política cultural brasileira neste momento propício de desenvolvimento e consolidação. Trazem referências, em termos de diretrizes, princípios e também de instrumentos de gestão, da mesma forma que a Convenção para Proteção e Promoção da Diversidade das Expressões Culturais serve de orientação política e conceitual para as definições de ações do poder público, iniciativa privada e sociedade civil. Ambos os documentos são parâmetros que devem orientar a construção do ethos do profissional da cultura, responsável por traduzir estes conceitos globais em práticas inovadoras, que assim fundamentadas podem apontar saídas efetivas para crescimento sustentável da humanidade.

\section{Considerações finais}

A estruturação do campo profissional da gestão cultural é ao mesmo tempo uma demanda e uma tendência latente em âmbito mundial.

A reflexão conceitual sobre as potencialidades e impactos da atuação do gestor da cultura nas diversas esferas societárias deve servir para fundamentar e orientar os profissionais da área em práticas que favoreçam a sustentabilidade, o desenvolvimento e os meios de expressão da sociedade.

Os projetos pedagógicos e para formação dos profissionais do setor devem 
considerar essa fundamentação teórica associada também a práticas eficientes favorecidas por ferramentas de gestão que precisam ser desenvolvidas. A transversalidade da área da cultura sugere possibilidades de reunir e consolidar conhecimentos sociais, artísticos administrativos, econômicos, ambientais criando uma dinâmica própria e profissional de atuação.

Favorecendo a presença da dimensão cultural nos processos de decisão em âmbito local, regional, nacional e internacional, se reforça uma potencialidade: construir no campo profissional da cultura novas relações e possíveis soluções para alguns impasses que rodeiam a sociedade contemporânea.

O atual contexto de estruturação das políticas públicas de cultura para o Brasil mostra-se favorável a esta proposta, uma vez que o poder público, com a participação da sociedade civil e instituições privadas, vêm criando novas condições para o desenvolvimento de ações culturais, sendo estratégica a participação de gestores culturais neste processo de construção e definição dos rumos da política cultural brasileira.

Desenvolver métodos e técnicas e alcançar uma nova compreensão do papel da cultura e, conseqüentemente, dos seus profissionais é um desafio do gestor cultural, que, por meio de sua atuação integral, pode apontar para novos paradigmas, métodos e sujeitos que sirvam à diversidade e possibilitem o alcance de formas alternativas e sustentáveis de relacionamento profissional na contemporaneidade.

\section{Referências bibliográficas}

AVELAR, Romulo. “O avesso da cena: notas sobre produção e gestão cultural”. Belo Horizonte: Duo editorial, 2008.

BARROS, José Márcio. "Diversidade cultural e gestão: apontamentos preliminares" in: "ENCONTRO DE ESTUDOS MULTIDISCIPLINARES EM CULTUR, 4, 2008", Salvador: CULT, 2008.

. "Diversidade Cultural: da proteção à promoção", Belo Horizonte: Autêntica Editora, 2008.

—. "Por uma cultura da avaliação da cultura". Disponível em: http:// www.itaucultural.org.br/bcodemidias/000978.pdf, acessado em 03/10/2009. 
CUNHA, Maria Helena; BERTELLI, Marcela. "Desafios para a formação de gestores da diversidade cultural" in: "ENCONTRO DE ESTUDOS MULTIDISCIPLINARES EM CULTURA, 4, 2008”. Salvador: CULT, 2008.

CUNHA, Maria Helena. “Gestão Cultural: profissão em formação”. Belo Horizonte. Duo Editorial, 2007.

"Os fazeres e os saberes dos gestores de cultura no Brasil" in: "Revista Observatório Itaú Cultural”, São Paulo: Itaú Cultural, n. 6, jul./set. 2008. Disponível em: http://www.itaucultural.org.br/bcodemidias/000991.pdf, acessado em 21/05/2009.

DEHEIZELIN, Lala. "Economia Criativa e empreendedorismo cultural" in: "ENCONTRO DE ESTUDOS MULTIDISCIPLINARES EM CULTURA, 2., 2006", Salvador: CULT, 2006.

DOWNING, John D. H. "Mídia Radical: rebeldia nas comunicações e movimentos sociais”, São Paulo: SENAC, 2002.

Fórum Mundial U-40. "Propostas para Diversidade Cultural 2030". Paris: UNESCO, junho de 2009. Disponível em: http://www.observatoriodadiversidade.org.br/ arquivos/u40_pt_br.pdf, acessado em 03/09/2009.

HALL, Stuart. "Da Diáspora: identidades e mediações culturais". Belo Horizonte: UFMG: Representações da UNESCO no Brasil, 2003. p. 254, 255.

Plano Nacional de Cultura. $2^{\mathrm{a}}$ edição [S.1: s/n], 2008.

RUBIM, Antonio Albino Canelas. "Formação em organização da cultura no Brasil" in: "Revista Observatório Itaú Cultural”, São Paulo: Itaú Cultural, n. 6, p. 47-55, jul./set. 2008.

SARAIVA, Enrique. "A Gestão da Cultura e a cultura da gestão: a importância da capacitação de administradores culturais" in: "ENCONTRO DE ESTUDOS MULTIDISCIPLINARES EM CULTURA, 4., 2008”. Salvador: CULT, 2008.

"Convenção sobre a Proteção e Promoção da Diversidade das Expressões Culturais", Paris: UNESCO, out. 2005. Disponível em: http://unesdoc.unesco.org/images/0014/ 001497/149742POR.pdf, acessado em15/09/2009. 